\title{
Geoinformation für jedermann - goldene Zeiten für die empirische Raumforschung
}

\author{
Ralf Bill
}

Online publiziert: 10. Mai 2014

(C) Springer-Verlag Berlin Heidelberg 2014

Räumliche Planung nutzt seit eh und je raumbezogene Informationen, früher in Form der analogen Karten, heute zunehmend sogenannte Geoinformationen in digitaler Form. Das Angebot an qualifizierten Geoinformationen hat sich im letzten Jahrzehnt einschneidend verbessert, seien dies Daten der öffentlichen Hand (z. B. zu Liegenschaften und zur Topographie aus der Vermessungsverwaltung, statistische Daten aus dem amtlichen Statistikbereich oder Umweltdaten aus der Umweltverwaltung), von kommerziellen Unternehmen (z. B. den Marktforschungsinstituten wie Infas 360 oder von GIS-Produktanbietern wie „Esri Maps and Data“) oder offene Daten (z. B. aus dem Vorhaben OpenStreetMap oder der OpenData-Initiative). Geoinformationen erreichen heute nicht mehr nur die fachlich versierten Nutzer in Planungsbüros oder Planungsverwaltungen, sondern auch den Bürger oder den politischen Entscheidungsträger. Spätestens seit Giganten wie Google, Apple oder Microsoft jedermann mit einem Blick auf sein lokales Umfeld mittels „Earth Viewern“/GeoBrowsern/Kartendiensten bedienen, sind Geoinformationen ins allgemeine Bewusstsein und in die ubiquitäre Nutzung gelangt. Und auch die Politik hat die Bedeutung des Themas für die Gesellschaft erkannt. Seit 2005 wird alle drei Jahre ein neuer Geo-Fortschrittsbericht der Bundesregierung publiziert, der 3. Geo-Fortschrittsbericht Ende 2012. Aktuell wird die Nationale Geoinformationsstrategie diskutiert.

Die zunehmend flächenhafte Verfügbarkeit von hochaufgelösten Geoinformationen hilft der empirischen Raumforschung, Prozesse in Stadt und Land besser zu verstehen, sie im Rechner nachzubilden, Szenarien durchzuspielen und

Prof. Dr. R. Bill ( $\square)$

Universität Rostock, Justus-von-Liebig-Weg 6,

18059 Rostock, Deutschland

E-Mail: ralf.bill@uni-rostock.de
Ergebnisse und Erkenntnisse dem Bürger oder Entscheider transparent und nachvollziehbar zu präsentieren. Die Kombinierbarkeit dieser unterschiedlichsten Geoinformationen über ihren Raumbezug in Geo-Informationssystemen (GIS) eröffnet ein Portfolio neuer analytischer Auswertungsmöglichkeiten. Hinzu kommen einfachere neue Erfassungsmethoden, z. B. durch massenhaft verteilte Sensorik (u. a. in den Smartphones, die beinahe jeder Bürger heute mit sich herumträgt) oder durch hochaufgelöste Fernerkundungsdaten (vom Satelliten mit unter $1 \mathrm{~m}$ Bodenauflösung bis zum Unmanned Aerial Vehicle (UAV) mit cm-Auflösung, vom optischen Wellenspektrum über Laser (Airborne oder terrestrisches Laserscanning) bis zu Radarwellen).

In diesem Schwerpunktheft greift die Zeitschrift „Raumforschung und Raumordnung“ diese Entwicklungen auf und präsentiert gesammelt Beiträge aus der räumlichen Forschung, in denen verfügbare Geoinformationen eine essenzielle Rolle spielen.

Der Beitrag von Stefan Fina, Angelika Krehl, Stefan Siedentop, Hannes Taubenböck und Michael Wurm demonstriert neue Möglichkeiten der Vernetzung von Geobasis-, Statistik- und Erdbeobachtungsdaten zur räumlichen Analyse und Visualisierung von Stadtstrukturen mit Dichteoberflächen. Hier werden am Beispiel der Stadt Köln aus Airborne-Laserscannerdaten Gebäudehöhen und Geschossigkeiten extrahiert, mit dem ATKIS-Basis-DLM kombiniert und auf Baublöcke aggregiert, für die dann blockspezifische Dichtekennwerte berechnet werden. Diese lassen sich anschließend mit statistischen Daten der Stadt Köln und disaggregierten Beschäftigungsdaten des „Instituts für Arbeitsmarkt- und Berufsforschung“ (IAB) verschneiden. Im Ergebnis entstehen räumliche Auswertungen und Visualisierungen, die Trends und Zustände von Dichteverteilungen über das Stadtgebiet veranschaulichen. 
Birgit Laggner, Natascha Orthen, Bernhard Osterburg und Norbert Röder gehen - anhand verschiedener hochaufgelöster Daten zur Landnutzung, zu Landnutzungsänderungen und Standortbedingungen (z. B. mittels Agrarstatistik, InVeKos, Basis-DLM, Daten zu Biogasanlagen) für Deutschland als Ganzes sowie mit einem höheraufgelösten Datenbestand für Niedersachsen in den Jahren 2005 bis 2007- der Frage nach, ob die zunehmende Biogasproduktion als Erklärung für den Grünlandschwund in Deutschland herhalten kann. Auf der Basis der Ergebnisse werden verschiedene Ansätze zum Schutz des Dauergrünlandes im Speziellen und zur Begrenzung von direktem und indirektem Landnutzungswandel im Allgemeinen diskutiert.

Jana Hoymann und Roland Goetzke stellen ein methodisches Framework vor, mit dem sich Szenarien der künftigen Landnutzung in Deutschland bis zum Jahr 2030 entwickeln lassen. Hierzu kommt ein rasterbasiertes Simulationsmodell zum Einsatz, das Daten unterschiedlicher räumlicher, zeitlicher und thematischer Auflösung und Skalenebenen konsistent zusammenführt. Der Fokus liegt dabei auf der Siedlungs- und Verkehrsflächenentwicklung. Im Ergebnis können sie Regionen mit künftig hoher Flächeninanspruchnahme - die großen Metropolen Hamburg, Berlin, München und Rhein-Main - und mit besonders geringer Flächeninanspruchnahme (Sachsen-Anhalt, Mecklenburg-Vorpommern sowie im Schwarzwald und der Schwäbischen Alb) identifizieren.

Mei-Ing Ruprecht wertet Forschungsarbeiten zu kleinräumigen baulich-räumlichen Daten für die Planung aus, in denen Fragestellungen zur Siedlungsentwicklung, Innenentwicklung und zu Veränderungen am Wohnungsmarkt eine Rolle spielen. Sie leitet daraus Anforderungen an die Daten ab und versucht, den Nutzen solcher hochaufgelösten Daten zu illustrieren. Mehrwerte sieht sie insbesondere in der Visualisierung (auch als Kommunikationsmittel), der kleinräumigen Analyse der baulich-räumlichen Siedlungsstruktur, der Verknüpfung anderer Daten mit der baulich-räumlichen Struktur (als Grundlage für bauliche Maßnahmen) und als Grundlage für überschlägige Berechnungen mittels Stadtstrukturtypen.

Die Raumordnung auf dem Meer gewinnt zunehmend an Bedeutung. Holger Janßen und Anne Hiller stellen das „Wadden Sea Region Planning Portal“ vor, ein reines Visualisierungsportal, das Akteuren die Partizipation an
Prozessen der Meeresraumordnung und anderen Meeresmanagementmaßnahmen erleichtert, indem es einen webbasierten Zugang zu grenzübergreifenden marinen Raumdaten in der Nordsee schafft. In ihrem Erfahrungsbericht beschreiben sie auch die Probleme und den Aufwand, mit denen sie bei der Erstellung einer hochwertigen Geodatenbasis mit über 100 harmonisierten Layern konfrontiert waren. Im Ergebnis können sie so mithilfe neuer Geodaten und internetbasierter Kartenanwendungen den Bedarfen und Problemen von Akteuren im Bereich der Meeresraumordnung begegnen.

In naher Zukunft werden verschiedene Probleme, die in den Beiträgen in der Nutzung von Geoinformationen zur Sprache kamen, auch behoben sein. Mit GovData (https:// www.govdata.de/), dem Datenportal für Deutschland, werden vermehrt Daten aller Verwaltungsebenen zentral und einfach zugänglich gemacht. Mit INSPIRE (Infrastructure for Spatial Information in Europe), einer gemeinsamen Geodateninfrastruktur in Europa, wird europaweit die nationale wie auch grenzübergreifende Nutzung einheitlich beschriebener und harmonisierter Geoinformationen und deren servicebasierte Bereitstellung über das Internet erleichtert. Somit ist zukünftig Beschaffung, Aufbereitung und Nutzung von Geoinformationen deutlich weniger aufwendig, als dies noch in manchen Beiträgen beschrieben ist. Der Gesetzgeber hat ebenfalls Defizite erkannt: So wird nach $\S 14$ des E-Government-Gesetzes (EGovG) die Georeferenzierung öffentlicher Register vorgeschrieben, im Bundesstatistikgesetz wird zukünftig auch ein Rasterdatensatz von mindestens $100 \mathrm{~m} \times 100 \mathrm{~m}$ Zellengröße erlaubt sein. Und auch an der Datenschutzfront fordert der 3. Geo-Fortschrittsbericht Auflösungsschwellen, um die Schutzbedürftigkeit aus datenschutzrechtlicher Sicht klarer zu regeln. Internationale Normen und Standards und deren konsequente Nutzung seitens der Softwareanbieter und der Datenbereitsteller sorgen zusätzlich dafür, dass die Interoperabilität der verteilten Systeme gewährleistet ist und der Nutzer - ob Wissenschaftler, Praktiker oder Laie - sich auf seine Fragestellung konzentrieren kann und sich nicht mehr mit technischen Problemen wie unterschiedlichen Koordinatenreferenzsystemen, vielfältigen Datenformaten und semantischem Interpretationsspielraum in den Informationen herumschlagen muss.

Goldene Zeiten also für die empirische Raumforschung. 